\title{
Comparison of Imaging Techniques for Tracking Cardiac Stem Cell Therapy
}

A growing number of exciting animal and preclinical studies are beginning to reveal the immense potential in stem cell-based therapies, particularly in the area of treating cardiovascular diseases. However, to evaluate the efficacy of these treatments in clinical trials, the transplanted stem cells must be monitored quantitatively and qualitatively in vivo. To date, several noninvasive imaging approaches have been used to follow the fate of stem cells in vivo. Here, we review the basic principles of the current techniques for cardiac stem cell tracking, compare the relative advantages and disadvantages of these imaging modalities, and discuss the future prospects for cardiac stem cell trafficking.

Despite recent advances in medical and surgical treatments, cardiovascular diseases remain the number one cause of morbidity and mortality in the United States. The societal and financial consequences are tremendous. For example, the American Heart Association estimates the economic costs of cardiovascular diseases in the United States for 2007 at $\$ 431.8$ billion, including direct and indirect costs incurred by cardiovascular diseases (1). In adult tissues such as those of the heart, the capacity for selfregeneration is limited. One promising approach is to inject into damaged hearts stem cells, which could potentially repopulate the myocardium, induce neovascularization, and lead to significant functional improvement (2). Encouraging animal studies from the late 1990s and early 2000s have led to the initiation or completion of several human clinical trials involving transplantation of bone marrow stem cells, skeletal myoblasts, or circulating progenitor cells into the heart (3). However, contradictory results from recent studies that used different origins of therapeutic stem cells and different routes of cell delivery highlight the need to elucidate the molecular mechanisms by which stem cells actually contribute to cardiac functional recovery (4). For instance, Lunde et al. showed that at 6 mo after intracoronary injection of mononuclear bone marrow cells into the infarcted heart, no significant improvement in left ventricular ejection fraction was observed (5). Similarly, Janssens et al. failed to detect any considerable improvement in ventricular function at 4 mo after injection of mononuclear bone marrow cells (6). By contrast, Schachinger

\footnotetext{
Received Jul. 30, 2007; revision accepted Sep. 6, 2007.

For correspondence or reprints contact: Joseph C. Wu, MD, PhD, Stanford University School of Medicine, 300 Pasteur Dr., Edwards Building, R354, Stanford, CA 94305-5344.

E-mail: joewu@stanford.edu

COPYRIGHT (c) 2007 by the Society of Nuclear Medicine, Inc.

DOI: 10.2967/jnumed.107.043299
}

et al. found that 59 patients with acute myocardial infarction who were treated with direct intracoronary infusion of either circulating progenitor cells or bone marrow-derived progenitor cells showed significant improvements in left ventricular ejection fraction and end-systolic volume (7). Clearly, these mixed results on cardiac stem cell therapy are both perplexing to scientists and frustrating to patients. Further studies are therefore urgently needed to clarify the discrepancies in clinical trials and validate the efficacy of cardiac repair using therapeutic stem cells.

\section{PRINCIPLES OF STEM CELL IMAGING}

The introduction of potentially therapeutic stem cells into patients requires concurrent techniques that provide noninvasive assessment of the survival, distribution, and differentiation of these cells. Molecular imaging, a multidisciplinary field that covers cell and molecular biology, imaging technology, and molecular medicine, provides integrated information on specific molecules of interest within the cells of living subjects and thus holds great promise as an effective way to track the transplanted cells. The unique information obtained from molecular imaging techniques is particularly helpful in evaluations of the functional outcomes of cell engraftment and may shed light on the mixed findings regarding cardiac stem cell-based therapy. The current noninvasive imaging approaches for tracking stem cells in vivo include imaging with magnetic particles, radionuclides, quantum dots (QDs), and reporter genes. A schematic of these approaches is shown in Figure 1. The ideal modality for tracking stem cells requires the imaging agents to be nontoxic, biocompatible, and highly specific to reduce perturbation to the target cells. In addition, the ideal imaging agents should be able to detect single cells and should show a decrease in signal with cell loss and an increase with cell proliferation. At this point, none of the extant imaging modalities has all these characteristics. In this review, we will highlight the current imaging modalities and evaluate their relative advantages and limitations.

\section{Magnetic Particle Labeling}

MRI is a widely used imaging modality for in vivo cell tracking in preclinical and clinical cases. Traditional MRI manipulates hydrogen nuclei in fluid under a static magnetic field to obtain regional contrasts based on differences in proton density, flow, or biochemical structure. To be tracked in infarcted myocardium, stem cells need to be enriched with a contrast agent that produces a sufficient 


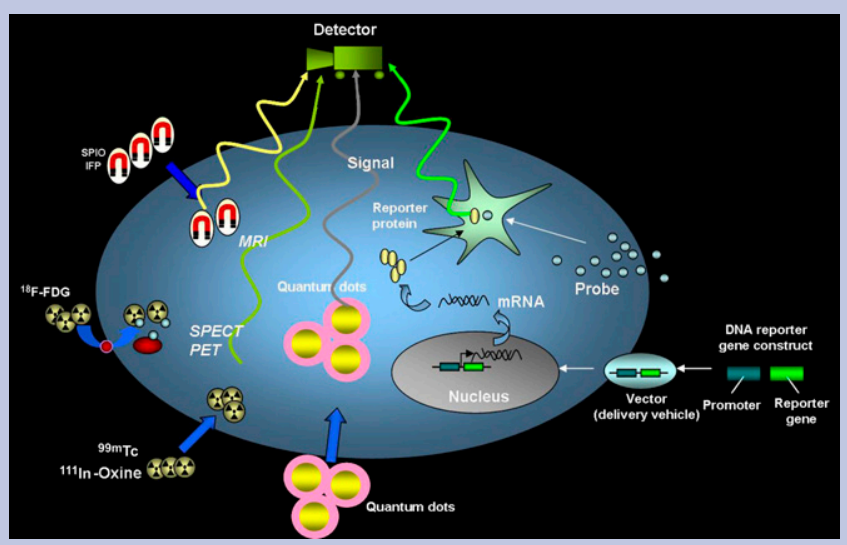

FIGURE 1. Schematic for noninvasive imaging of stem cell fate in myocardium. The 4 different techniques include magnetic particle labeling, radionuclide labeling, QD labeling, and reporter gene labeling. SPIO = superparamagnetic iron oxide; IFP = iron fluorescent particles.

positive or negative signal to distinguish them from the background. Two major classes of contrast imaging materials have been developed. One uses lanthanide gadolinium $\left(\mathrm{Gd}^{3+}\right)$ to generate signals on T1-weighted contrastenhanced images. The other type uses superparamagnetic iron oxide particles to generate signals on T2-weighted images. Several studies have demonstrated that incorporation of a gadolinium-based contrast agent into stem cells allows the tracking of magnetically labeled cells for up to 6 wk (8). However, at present, superparamagnetic iron oxide particles are the preferred agent for cell tracking because they are capable of 3-dimensional imaging at low concentrations $(5-10 \mu \mathrm{m} / \mathrm{L})(9)$. The sensitivity of SPIO is achieved through a large dipolar magnetic field gradient experienced by protons near the particles. The detectability of labeled cells also depends on the number of cells injected into the myocardium. For instance, $10^{7}-10^{8}$ SPIO-labeled mesenchymal stem cells can readily be detected by MRI when injected directly into the myocardium of a swine, as shown in Figure 2 (10). Using an SPIO contrast agent, Kraitchman et al. successfully demonstrated long-term evaluation of magnetically labeled mesenchymal stem cells after myocardial infarction in a swine model (11). The same group later used SPIO colabeled with ${ }^{111} \mathrm{In}$-oxine to compare the relative sensitivities of MRI versus radionuclide imaging and observed that SPECT/CT was more sensitive than MRI for detecting cell homing in the heart (12). Furthermore, intrinsic problems exist with MRI contrast agents, including the difficulty of quantification with cell division and possible transfers of contrast agents to neighboring cells. Another limitation with MRI is the contraindications for patients with implantable devices (e.g., pacemakers and defibrillators), who often are the very patients with refractory symptoms requiring novel stem cell therapy.

\section{Radionuclide Labeling}

Radionuclide imaging techniques, including PET and SPECT, have garnered intense research interest over the
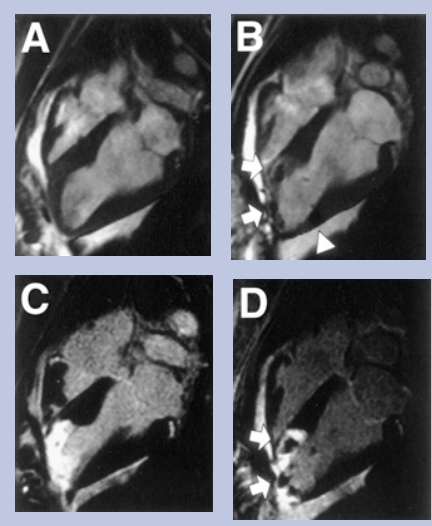

FIGURE 2. MRI of transplanted cardiac stem cells in pigs. (A and $B$ ) Long-axis view of left ventricle before $(A)$ and after (B) transcatheter injection of $4 \times 10^{6}$ iron fluorescent particle (IFP)-labeled mesenchymal stem cells (MSCs) into infarct at apex (arrows) and into adjoining normal myocardium (arrowhead). (C and D) Long-axis view with delayed, hyperenhanced inversion recovery highlights areas of nonviable infarcted myocardium before (C) and after (D) injection of IFP-labeled MSCs. MSCs appear dark against hyperenhanced infarct. (Reprinted with permission from (10).)

past decade. Compared with MRI, PET and SPECT provide high intrinsic sensitivity and can use a variety of clinically tested imaging agents. Recent improvements in spatial resolution (1-2 $\mathrm{mm})$ have made radionuclide imaging particularly suitable for cell tracking (13). Direct labeling of cells with radioisotopes in clinical practice has used ${ }^{111} \mathrm{In}$-oxyquinoline and ${ }^{99 \mathrm{~m}} \mathrm{Tc}$-hexamethylpropylene amine oxime. Labeling cells with ${ }^{111} \mathrm{In}$-oxyquinoline is a wellestablished method that has been deployed in endothelial progenitor cells, hematopoietic stem cells, and mesenchymal stem cells $(14,15)$. For instance, using ${ }^{111}$ In-radiotracer, Aicher et al. observed that only $4.7 \%$ of the injected endothelial and hematopoietic progenitor cells were retained in the infarcted myocardium (15). A similar observation of cell targeting was found using ${ }^{99 \mathrm{~m}} \mathrm{Tc}$-labeled mesenchymal stem cells (16). Furthermore, in the first human study, the ${ }^{18} \mathrm{~F}-$ FDG PET radiotracer was used to follow the intracoronary delivery of bone marrow cells in patients after myocardial infarction (17). These investigators found that $14 \%-39 \%$ of CD34-positive cells accumulated in the infarcted myocardium approximately $1 \mathrm{~h}$ after intracoronary delivery (Fig. 3).

Although nuclear imaging has major advantages, it is limited by concerns such as the potential transfer of radiotracer to nontargeted cells and potential adverse effects of the radiotracer on stem cell viability, function, and differentiation capacity. For instance, a ${ }^{111}$ In-labeled radiotracer has been shown to impair proliferation and differentiation in CD34-positive hematopoietic progenitor cells (18). Additional studies thus are needed to examine the effects of labeling on the capacity to differentiate stem cells of various origins.

\section{Nanoparticle Labeling}

Semiconductor QDs are a new class of fluorescent probes that has been used in noninvasive imaging in recent years $(19,20)$. This technique makes use of fluorescent semiconductor nanocrystals to detect membrane molecules of interest. The excitation wavelengths of the QDs can be manipulated and range from ultraviolet to nearly infrared. Depending on the 
FIGURE 3. Evaluation of myocardial homing and biodistribution of ${ }^{18}$ F-FDGlabeled bone marrow cells after intracoronary delivery in patient after myocardial infarction. (A and B) Representative left posterior oblique $(A)$ and left anterior oblique $(B)$ views at $65 \mathrm{~min}$ after injection of ${ }^{18} \mathrm{~F}-\mathrm{FDG}-$ labeled unselected bone marrow cells into the left circumflex coronary artery.
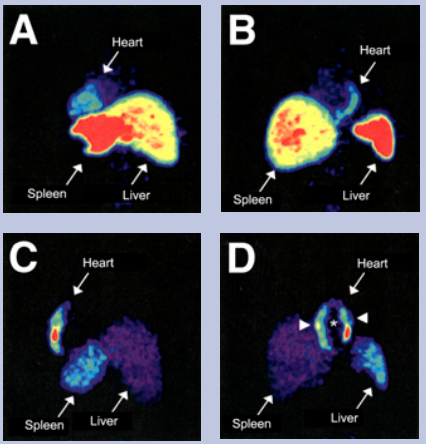

(C and D) Representative left posterior oblique (C) and left anterior oblique (D) views at 70 min after injection of ${ }^{18} \mathrm{~F}-\mathrm{FDG}$-labeled CD34-positive subpopulation of bone marrow cells into left anterior descending coronary artery. (Reprinted with permission from (17).)

size and composition of these probes, they can be designed to emit different wavelengths of light. The photostability of QDs, as seen in their resistance to photobleaching and longlasting fluorescence, makes them appealing for tracking stem cells in vivo (21). However, the effects of QDs on stem cell proliferation and differentiation remain unclear because mixed results have been reported using different origins of stem cells or experimental protocols $(19,22)$. Finally, several other obstacles, including the tendency for aggregation of QDs in the cytosol, difficulty with the delivery of QDs into cells, and nonspecific binding to multiple molecules (23), must be overcome before QDs can realize their full potential in clinical imaging.

\section{Reporter Gene Labeling}

Reporter gene imaging has emerged as a unique tool for localizing specific molecules of interest within living subjects. The reporter gene of interest usually encodes a specific protein that, when it interacts with an imaging probe, generates some form of signal that can be captured and quantified by an imaging modality such as MRI, PET, SPECT, or an optical charge-coupled device. Several studies have successfully demonstrated tracking of the distribution and engraftment of stem cells in mice and rats using bioluminescence-based optical imaging $(24,25)$. Although the signals generated by firefly luciferase are more sensitive than those of other imaging modalities in small animals, luciferase proteins and their substrates have a high absorption rate in hemoglobin and can easily be scattered within deep tissues. For now, this current inability to accurately determine cell depth is a major limitation.

Fluorescence imaging, another widely used type of optical imaging, applies a fluorescent protein for in vivo imaging. Compared with bioluminescence, near-infrared fluorescence imaging has reduced absorption and scatter of photons at $700-1,000 \mathrm{~nm}$. However, similar to bioluminescence, near-infrared fluorescence imaging is restricted to a shallow tissue depth (between 4 and $10 \mathrm{~cm}$ ). In addition to the bioluminescence and fluorescence reporter genes, other types of reporter genes are available, including herpes simplex virus thymidine kinase for PET $(24,26)$ (Fig. 4), sodium iodide symporter for SPECT (27), and transferrin receptor for MRI (28).

Applying the concept of reporter gene labeling has a significant potential to furnish greater insights into the mechanisms of stem cell therapy. For instance, inducible promoters can be used to modulate the level of reporter gene expression and therapeutic gene expression (29). Tissue-specific promoters may be used as sensors of the cell differentiation state. In this approach, a cardiac-specific promoter-such as $\alpha$-cardiac myosin heavy-chain promoter-driving the expression of an antibiotic resistance gene can be used to

FIGURE 4. Tracking of transplanted embryonic stem cells in rats by multimodality molecular imaging. (A) Bioluminescence imaging (top) and small-animal PET (bottom) of embryonic stem cell fate in living animals. Representative images demonstrate imaging signal activities in these mouse embryonic stem cells that stably express firefly luciferase (bioluminescence imaging), monomeric red fluorescence protein (fluorescence), and herpes simplex virus truncated thymidine kinase (PET) triple-fusion reporter gene. (B) Significant increase of firefly luciferase and truncated thymidine kinase activities were seen from week 2 to week 4 , indicative of in vivo teratoma formation. (C) In vivo correlation between bioluminescence and PET imaging signals. $\mathrm{BLI}=$ bioluminescence imaging; $\mathrm{d}=$ day; $\mathrm{P}=$ photons; $\mathrm{sr}=$ steridian; $\mathrm{tTk}$ $=$ truncated thymidine kinase; $\mathrm{w}=$ week. (Reprinted with permission from (24).)
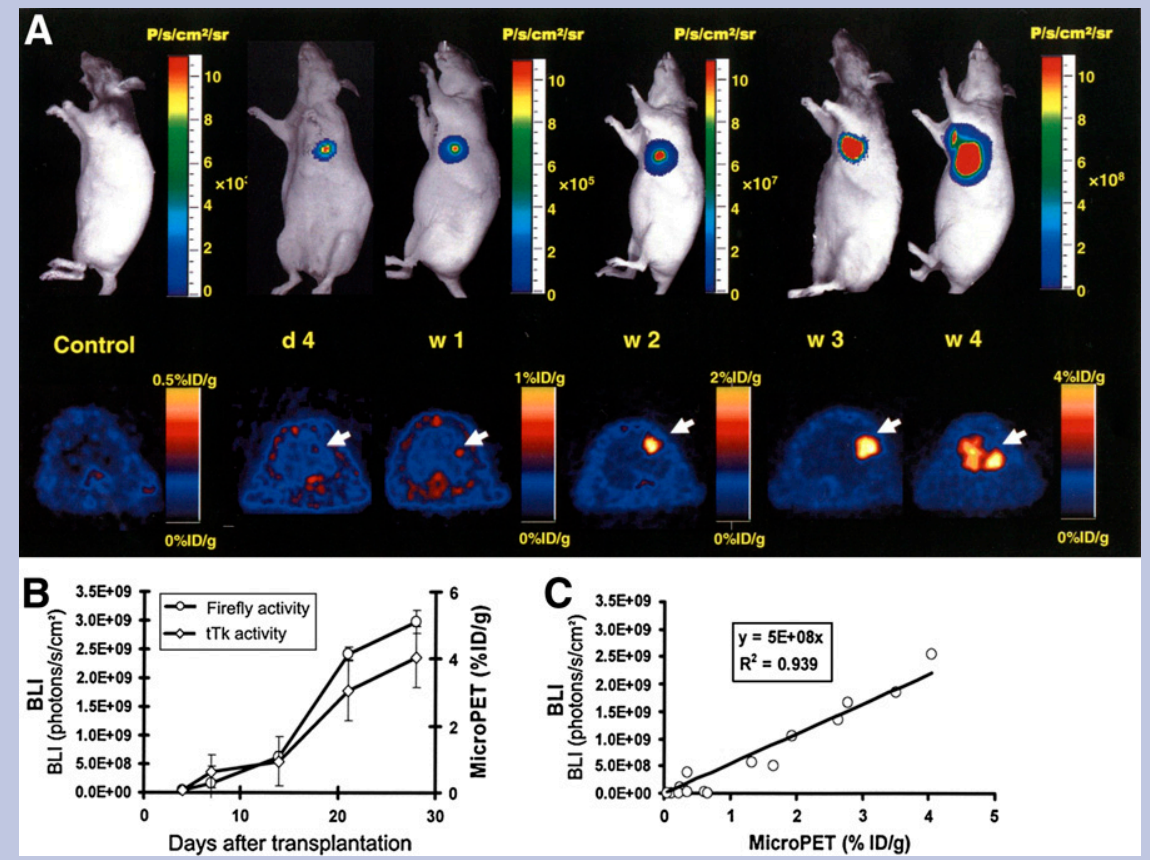
select for cardiomyocytes in the mixed culture (30). Development of a similar system for monitoring stem cell differentiation in vivo using firefly luciferase (bioluminescence) or herpes simplex virus thymidine kinase (PET) would yield obvious benefits. However, several problems must be solved before reporter gene imaging can fully and safely be applied clinically. These issues include finding appropriate probes that elicit minimal or no immunogenic response, enhancing transfection stability, and minimizing the potential interference of the stem cell function and differentiation from vector transfection or transduction.

\section{FUTURE PERSPECTIVES}

Stem cell tracking requires high sensitivity and high spatial resolution. Currently, no imaging modality is perfect in all aspects. Although MRI provides a safe profile and 3 -dimensional capacity, it has the lowest sensitivity $\left(10^{-3}\right.$ to $10^{-5} \mathrm{~mol} / \mathrm{L}$ ) and is contraindicated in patients with implantable devices. Radiolabeling imaging has a fair sensitivity $\left(10^{-8}\right.$ to $\left.10^{-9} \mathrm{~mol} / \mathrm{L}\right)$ but is not suitable for long-term cell tracking because of radioisotope decay. QDs provide superior photostability and long-term multicolor in vivo imaging, but concerns such as possible toxicity to cells and dispersals of QDs in the cytosol need to be resolved. Bioluminescence $\left(10^{-15}\right.$ to $\left.10^{-17} \mathrm{~mol} / \mathrm{L}\right)$ and near-infrared fluorescence $\left(10^{-9}\right.$ to $\left.10^{-12} \mathrm{~mol} / \mathrm{L}\right)$ imaging are the most sensitive among the available imaging modalities, but both techniques are constrained to a relatively shallow tissue depth. Reporter gene imaging, though able to assess cell fate (viability and proliferation) more accurately, will need to demonstrate cell safety because of the genetic modification.

Given that each imaging modality presents its unique set of advantages and drawbacks, one should ask what biologic questions need to be answered before choosing an appropriate imaging modality. If the exact location of cell delivery must be identified, then MRI is the preferred choice. If only the short-term fate of transplanted cells needs to be determined, then radiolabeling techniques are suitable. If knowledge of the long-term fate of transplanted stem cells is desired, then reporter gene imaging is best. Finally, future efforts should also focus on multimodality imaging approaches, which may minimize the potential drawbacks of using each imaging modality alone, as it is possible that a tailored combination of 2 or more techniques may provide the most ideal information profile for clinical applications.

\section{Sarah J. Zhang Joseph C. Wu Stanford University School of Medicine Stanford, California}

\section{REFERENCES}

1. Rosamond W, Flegal K, Friday G, et al. Heart disease and stroke statistics: 2007 update - a report from the American Heart Association Statistics Committee and Stroke Statistics Subcommittee. Circulation. 2007;115:e69-e171.
2. Orlic D, Kajstura J, Chimenti S, et al. Bone marrow cells regenerate infarcted myocardium. Nature. 2001;410:701-705.

3. Wollert KC, Drexler H. Clinical applications of stem cells for the heart. Circ Res. 2005;96:151-163.

4. Rosenzweig A. Cardiac cell therapy: mixed results from mixed cells. $N$ Engl J Med. 2006;355:1274-1277.

5. Lunde K, Solheim S, Aakhus S, et al. Intracoronary injection of mononuclear bone marrow cells in acute myocardial infarction. $N$ Engl J Med. 2006;355: 1199-1209.

6. Janssens S, Dubois C, Bogaert J, et al. Autologous bone marrow-derived stemcell transfer in patients with ST-segment elevation myocardial infarction: double-blind, randomised controlled trial. Lancet. 2006;367:113-121.

7. Schachinger V, Assmus B, Britten MB, et al. Transplantation of progenitor cells and regeneration enhancement in acute myocardial infarction: final one-year results of the TOPCARE-AMI Trial. J Am Coll Cardiol. 2004;44:1690-1699.

8. Kim D, Hong KS, Song J. The present status of cell tracking methods in animal models using magnetic resonance imaging technology. Mol Cells. 2007;23:132137.

9. Rogers WJ, Meyer CH, Kramer CM. Technology insight: in vivo cell tracking by use of MRI. Nat Clin Pract Cardiovasc Med. 2006;3:554-562.

10. Hill JM, Dick AJ, Raman VK, et al. Serial cardiac magnetic resonance imaging of injected mesenchymal stem cells. Circulation. 2003;108:1009-1014.

11. Kraitchman DL, Heldman AW, Atalar E, et al. In vivo magnetic resonance imaging of mesenchymal stem cells in myocardial infarction. Circulation. 2003; 107:2290-2293.

12. Kraitchman DL, Tatsumi M, Gilson WD, et al. Dynamic imaging of allogeneic mesenchymal stem cells trafficking to myocardial infarction. Circulation. 2005; 112:1451-1461.

13. Acton PD, Kung HF. Small animal imaging with high resolution single photon emission tomography. Nucl Med Biol. 2003;30:889-895.

14. Berman DS, Hachamovitch R, Shaw LJ, et al. Roles of nuclear cardiology, cardiac computed tomography, and cardiac magnetic resonance: assessment of patients with suspected coronary artery disease. J Nucl Med. 2006;47:74-82.

15. Aicher A, Brenner W, Zuhayra M, et al. Assessment of the tissue distribution of transplanted human endothelial progenitor cells by radioactive labeling. Circulation. 2003;107:2134-2139.

16. Barbash IM, Chouraqui P, Baron J, et al. Systemic delivery of bone marrowderived mesenchymal stem cells to the infarcted myocardium: feasibility, cell migration, and body distribution. Circulation. 2003;108:863-868.

17. Hofmann M, Wollert KC, Meyer GP, et al. Monitoring of bone marrow cell homing into the infarcted human myocardium. Circulation. 2005;111:2198-2202.

18. Brenner W, Aicher A, Eckey T, et al. ${ }^{111}$ In-labeled CD34+ hematopoietic progenitor cells in a rat myocardial infarction model. $J$ Nucl Med. 2004;45:512-518.

19. Dubertret B, Skourides P, Norris DJ, Noireaux V, Brivanlou AH, Libchaber A. In vivo imaging of quantum dots encapsulated in phospholipid micelles. Science. 2002;298:1759-1762.

20. Jaiswal JK, Mattoussi H, Mauro JM, Simon SM. Long-term multiple color imaging of live cells using quantum dot bioconjugates. Nat Biotechnol. 2003;21:47-51.

21. Lin S, Xie X, Patel MR, et al. Quantum dot imaging for embryonic stem cells. BMC Biotechnol. 2007;7(1):67 [Epub ahead of print].

22. Hsieh SC, Wang FF, Lin CS, Chen YJ, Hung SC, Wang YJ. The inhibition of osteogenesis with human bone marrow mesenchymal stem cells by $\mathrm{CdSe} / \mathrm{ZnS}$ quantum dot labels. Biomaterials. 2006;27:1656-1664.

23. Jaiswal JK, Simon SM. Potentials and pitfalls of fluorescent quantum dots for biological imaging. Trends Cell Biol. 2004;14:497-504.

24. Cao F, Lin S, Xie X, et al. In vivo visualization of embryonic stem cell survival, proliferation, and migration after cardiac delivery. Circulation. 2006;113:10051014.

25. Sheikh AY, Lin SA, Cao F, et al. Molecular imaging of bone marrow mononuclear cell homing and engraftment in ischemic myocardium. Stem Cells. 2007;25:2677-2684.

26. Wu JC, Chen IY, Sundaresan G, et al. Molecular imaging of cardiac cell transplantation in living animals using optical bioluminescence and positron emission tomography. Circulation. 2003;108:1302-1305.

27. Miyagawa M, Beyer M, Wagner B, et al. Cardiac reporter gene imaging using the human sodium/iodide symporter gene. Cardiovasc Res. 2005;65:195-202.

28. Weissleder R, Moore A, Mahmood U, et al. In vivo magnetic resonance imaging of transgene expression. Nat Med. 2000;6:351-355.

29. Xie X, Cao F, Sheikh AY, et al. Genetic modification of embryonic stem cells with VEGF enhances cell survival and improves cardiac function. Cloning Stem Cells. 2007. In press.

30. Klug MG, Soonpaa MH, Koh GY, Field LJ. Genetically selected cardiomyocytes from differentiating embryonic stem cells form stable intracardiac grafts. J Clin Invest. 1996;98:216-224. 